\title{
THE $l_{1}$-COMPLETION OF A METRIC COMBINATORIAL $\infty$-MANIFOLD
}

\author{
KATSURO SAKAI
}

\begin{abstract}
Let $K$ be a simplicial complex. The realization $|K|$ of $K$ admits the metric

$$
d_{1}(x, y)=\sum_{v \in K^{0}}|x(v)-y(v)|
$$

where $x(v)$ and $y(v), v \in K^{0}$, are the barycentric coordinates of $x$ and $y$ respectively. The completion of the metric space $\left(|K|, d_{1}\right)$ is called the $l_{1}$ completion and is denoted by $\overline{|K|}^{l_{1}}$. In this paper, we prove that $\overline{|K|}^{l_{1}}$ is an $l_{2}$-manifold if and only if $K$ is a combinatorial $\infty$-manifold.
\end{abstract}

0. Introduction. Let $K$ be a simplicial complex. The realization $|K|$ of $K$ admits the metric

$$
d_{1}(x, y)=\sum_{v \in K^{0}}|x(v)-y(v)|,
$$

where $x(v)$ and $y(v), v \in K^{0}$, are the barycentric coordinates of $x$ and $y$ respectively. The topology induced by $d_{1}$ is the metric topology of $|K|$ and the space $|K|$ with this topology is denoted by $|K|_{m}$. The completion of the metric space $\left(|K|, d_{4}\right)$ is called the $l_{1}$-completion of $|K|_{m}$ and is denoted by $\overline{|K|}^{l_{1}}$. In $\left[\mathbf{S a}_{1}\right]$, the author proved that $\overline{|K|}^{l_{1}}$ is an ANR and the inclusion $i:|K|_{m} \subset \overline{|K|}^{l_{1}}$ is a fine homotopy equivalence, that is, for each open cover $U$ of $\overline{|K|}^{l_{1}}$ there is a map $f: \overline{|K|}^{l_{1}} \rightarrow|K|_{m}$ such that $i \circ f$ is $\mathcal{U}$-homotopic to id and $f \circ i$ is $i^{-1}(\mathcal{U})$-homotopic to id, and conjectured that $\overline{|K|}^{l_{1}}$ is an $l_{2}$-manifold if $K$ is a combinatorial $\infty$-manifold. Here a combinatorial $\infty$-manifold is a countable simplicial complex such that the star of each vertex is combinatorially equivalent to the countable-infinite full simplicial complex, namely a $\infty$-simplex $\Delta^{\infty}$ (see [Sa $\left.\mathbf{S a}_{1}\right]$ ). An $l_{2}$-manifold is a separable manifold modeled on the Hilbert space $l_{2}$. Let $l_{2}^{f}$ be the linear span of the natural orthonormal basis of $l_{2}$. A separable manifold modeled on the space $l_{2}^{f}$ is called an $l_{2}^{f}$-manifold. In $\left[\mathbf{S a}_{2}, \mathbf{S a}_{3}\right]$, it was shown that $K$ is a combinatorial $\infty$-manifold if and only if $|K|_{m}$ is an $l_{2}^{f}$-manifold. The main result of this paper is the following

MAIN THEOREM. A simplicial complex $K$ is a combinatorial $\infty$-manifold if and only if $\overline{|K|}^{l_{1}}$ is an $l_{2}$-manifold.

1. Preliminaries. Let $X$ be a metric space with a metric $d$. A closed subset $A$ of $X$ is called a $Z$-set in $X$ if for each $\varepsilon>0$ and each map $f: I^{n} \rightarrow X, n \in \mathrm{N}$,

Received by the editors May 21, 1986.

1980 Mathematics Subject Classification (1985 Revision). Primary 57N20, 57Q15, 54E52.

Key words and phrases. Simplicial complex, combinatorial $\infty$-manifolds, the metric topology, completion, $l_{2}^{f}$-manifold, $l_{2}$-manifold, $Q$-manifold, (f.d.) cap set, $Z$-set. 
there is a map $g: I^{n} \rightarrow X \backslash A$ with $d(f, g)<\varepsilon$. An (f.d.) cap set for $X$ is a subset $M$ of $X$ such that $M=\bigcup_{n=1}^{\infty} M_{n}$ where $M_{1} \subset M_{2} \subset \cdots$ is a tower of (finite-dimensional) compact $Z$-sets in $X$ such that for each $\varepsilon>0$, each $m \in \mathbf{N}$, and each (finite-dimensional) compact subset $A$ of $X$, there is an $n \in \mathbf{N}$ and an embedding $h: A \rightarrow M_{n}$ such that $h \mid A \cap M_{m}=$ id and $d(h$,id $)<\varepsilon$. Let $s$ denote the pseudo-interior of the Hilbert cube $Q=I^{\omega}$, that is, $s=I^{\omega}$, where $I=[0,1]$ and $\stackrel{\circ}{I}=(0,1)$. The pseudo-boundary $Q \backslash s$ is a cap set for $Q$. (Refer to [An] and $\left[\mathrm{Ch}_{1}\right]$.) A $Q$-manifold is a separable manifold modeled on the Hilbert cube $Q$. T. A. Chapman $\left[\mathbf{C h}_{1}\right]$ proved that if $M$ is a cap set for a $Q$-manifold $X$, then $X \backslash M$ is an $l_{2}$-manifold.

1.1 LEMMA. Let $X$ be a locally compact ANR with a metric $d$ and let $M=$ $\bigcup_{n=1}^{\infty} M_{n} \subset X$ such that each $M_{n}$ is a $Q$-manifold and a $Z$-set in $M_{n+1}$, and

for each $\varepsilon>0$, each $m \in \mathbf{N}$, and each compact subset $A$ of $X$, there is an $n \in \mathrm{N}$ and a map $f: A \rightarrow M_{n}$ such that $f\left(A \cap M_{m}\right) \subset M_{m}$ and $d(f, \mathrm{id})<\varepsilon$.

Then $X$ is a $Q$-manifold and $M$ is a cap set for $X$, hence $X \backslash M$ is an $l_{2}$-manifold.

Proof. It is easy to see that $X$ is a $Q$-manifold by using Torunczyk's characterization of $Q$-manifolds [To]. Since each compact set in a $Q$-manifold has a compact $Q$-manifold neighborhood (this can be easily seen by using $\left[\mathbf{C h}_{2}\right.$, Theorems 37.2 and 28.1]), we can write $M_{n}=\bigcup_{i=1}^{\infty} M_{n, i}, n \in \mathrm{N}$, where each $M_{n, i}$ is a compact $Q$-manifold, and

$$
M_{n, i} \subset \operatorname{int}_{M_{n}} M_{n, i+1} \cap \operatorname{int}_{M_{n+1}} M_{n+1, i} .
$$

Then observe that $M=\bigcup_{n=1}^{\infty} M_{n, n}$. Since $M_{n}$ is a $Z$-set in $M_{m}$ for any $m>n$, it follows from (*) that $M_{n, n}$ is a $Z$-set in $X$. Let $A$ be a compact subset of $X$ and let $\varepsilon>0$ and $m \in \mathbf{N}$. Since $M_{m}$ is an ANR, there is a $\delta>0$ such that any map $f: A \cap M_{m, m} \rightarrow M_{m}$ with $d(f$, id $)<\delta$ is $\varepsilon / 3$-homotopic to the inclusion $A \cap M_{m, m} \subset M_{m}$. By (*), we have a map $f: A \rightarrow M_{n^{\prime}}$ for some $n^{\prime}>m$ such that $f\left(A \cap M_{m}\right) \subset M_{m}$ and $d(f$, id $)<\delta$. Then $f \mid A \cap M_{m, m}$ is $\varepsilon / 3$-homotopic to the inclusion $A \cap M_{m, m} \subset M_{m}$. Since $M_{n^{\prime}}$ is an ANR, we have a map $g: A \rightarrow M_{n^{\prime}}$ such that $g \mid A \cap M_{m, m}=$ id and $d(g, f)<\varepsilon / 3$ by the Homotopy Extension Theorem $[\mathbf{H u}$, Chapter IV, Theorem 2.2 and its proof]. From compactness of $g(A), g(A) \subset M_{n^{\prime}, n}$ for some $n \geq n^{\prime}$ because

$$
M_{n^{\prime}}=\bigcup_{i=1}^{\infty} \operatorname{int}_{M_{n^{\prime}}} M_{n^{\prime}, i} .
$$

Then we have $g(A) \subset M_{n, n}$. Since $M_{m}$ is a $Z$-set in $M_{n}$ and

$$
A \cap M_{m, m} \subset M_{m} \cap \operatorname{int}_{M_{n}} M_{n, n},
$$

$A \cap M_{m, m}$ is a $Z$-set in $M_{n, n}$. By the $Z$-Embedding Approximation Theorem $\left[\mathrm{Ch}_{2}\right.$, Lemma 18.1], we have an embedding $h: A \rightarrow M_{n, n}$ such that $h \mid A \cap M_{m, m}=$ $g \mid A \cap M_{m, m}=$ id and $d(h, g)>\varepsilon / 3$, hence $d(h$, id $)<\varepsilon$. Therefore $M=\bigcup_{n=1}^{\infty} M_{n, n}$ is a cap set for $X$. 
2. Proof of the Main Theorem. By $S_{1}^{+}$, we denote the set of all points in the unit sphere of the Banach space $l_{1}$ having nonnegative coordinates, that is,

$$
S_{1}^{+}=\left\{\left(x_{i}\right)_{i \in \mathbf{N}} \mid \sum_{i=1}^{\infty} x_{i}=1 \text { and } x_{i} \geq 0 \text { for all } i \in \mathbf{N}\right\} .
$$

Clearly $S_{1}^{+} \subset Q$ as sets. It is well known and easy to see that the topology on $S_{1}^{+}$ induced by the norm $\|\cdot\|_{1}$ of $l_{1}$ coincides with the product topology. Thus $S_{1}^{+} \subset Q$ as topological spaces.

Let $K$ be a countable simplicial complex with vertices $v_{i}, i \in \mathbf{N}$. Identifying $x \in|K|$ with $\left(x_{i}\right)_{i \in \mathbf{N}}=\left(x\left(v_{i}\right)\right)_{i \in \mathbf{N}} \in S_{1}^{+}$, we consider $|K|_{m} \subset S_{1}^{+} \subset Q$. The $l_{1^{-}}$ completion $\overline{|K|}^{l_{1}}$ of $|K|_{m}$ is $\mathrm{cl}_{S_{1}^{+}}|K|$ because $d_{1}$ is the metric induced by the norm $\|\cdot\|_{1}$ and $S_{1}^{+}$is closed in $l_{1}$. On the other hand $|K|_{m}$ has the local-compactification

$$
\overline{|K|}^{Q^{*}}=\operatorname{cl}_{Q \backslash\{0\}}|K|=\left(\operatorname{cl}_{Q}|K|\right) \backslash\{0\},
$$

where 0 denotes the point $(0,0, \ldots) \in Q$. Then $\overline{|K|}^{l_{1}}$ is a subspace of $\overline{|K|}^{Q^{*}}$. Moreover, by [Sa $\mathbf{S a}_{4}$, Lemma 1.1; $\mathbf{S a} \mathbf{a}_{5}$, Lemma 1.1], we have the following

2.1 LEMMA. For a countable simplicial complex $K$ with no principal (maximal) simplex,

$$
\overline{|K|}^{Q^{*}}=(0,1] \cdot \overline{|K|}^{l_{1}}=\left\{t x \mid x \in \overline{|K|}^{l_{1}}, t \in(0,1]\right\}
$$

In $\left[\mathbf{S a}_{5}\right]$, the author proved that for a combinatorial $\infty$-manifold $K, \overline{|K|}^{Q^{*}}$ is a $[0,1)$-stable $Q$-manifold containing $|K|_{m}$ as an f.d. cap set. Here we say that a $Q$-manifold $X$ is $[0,1)$-stable if $X \times[0,1)$ is homeomorphic to $X$. Now we prove moreover that $\overline{|K|}^{Q^{*}} \sqrt{|K|^{l}}{ }^{l_{1}}$ is a cap set for $\overline{|K|}^{Q^{*}}$.

2.2 THEOREM. If $K$ is a combinatorial $\infty$-manifold, then $\overline{|K|}^{Q^{*}}$ is a $[0,1)$ stable $Q$-manifold, $|K|_{m}$ is an f.d. cap set for $\overline{|K|}^{Q^{*}}$, and $\overline{|K|}^{Q^{*}} \overline{|K|}^{l_{1}}$ is a cap set for $\overline{|K|}^{Q^{*}}$.

PrOOF. As mentioned above, the first two properties have been proved in $\left[\mathbf{S a}_{5}\right]$. To see the last property, we use Lemma 1.1. From 2.1, it follows that

$$
\overline{|K|}^{Q^{*}} \sqrt{|K|^{l}}=\bigcup_{n=1}^{\infty}\left(1-\frac{1}{n}\right) \cdot \overline{|K|}^{Q^{*}} \text {. }
$$

Each $(1-1 / n) \cdot \overline{|K|}^{Q^{*}}$ is a $Q$-manifold because it is homeomorphic to $\overline{|K|}^{Q^{*}}$. To see that $(1-1 / n) \cdot \overline{|K|}^{Q^{*}}$ is a $Z$-set in $(1-1 /(n+1)) \cdot \mid \bar{K}^{Q^{*}}$, it suffices to see that $t \cdot \overline{|K|}^{Q^{*}}$ is a $Z$-set in $\overline{|K|}^{Q^{*}}$ for $0<t<1$. In the proof of the Main Theorem of $\left[\mathbf{S a}_{5}\right]$, it is proved that for each compact subset $A$ of $\overline{|K|}^{Q^{*}}$ and each $\varepsilon>0$, there is a map $f: A \rightarrow|K|_{m}$ with $d_{Q}(f$, id $)<\varepsilon$, where $d_{Q}$ is the metric for $Q$ defined by

$$
d_{Q}(x, y)=\sup _{i \in \mathbf{N}} \min \left\{\left|x_{i}-y_{i}\right|, i^{-1}\right\}
$$


For $0<t<1,|K| \cap t \cdot \overline{|K|}^{Q^{*}}=\varnothing$. Then it follows that $t \cdot \overline{|K|}^{Q^{*}}$ is a $Z$-set in $\overline{|K|}^{Q^{*}}$. Condition $(*)$ of Lemma 1.1 is clearly satisfied. Then the result follows from Lemma 1.1.

Proof of The MAIN TheOREM. From the above theorem, it follows that if $K$ is a combinatorial $\infty$-manifold, then $\overline{|K|}^{l_{1}}$ is an $l_{2}$-manifold. Conversely assume that $\overline{|K|}^{l_{1}}$ is an $l_{2}$-manifold. Let $A$ be a simplex of $K$. Since any compact subset of an $l_{2}$-manifold is a $Z$-set, $A$ is a $Z$-set in $\overline{|K|}^{l_{1}}$. By [Sa $\mathbf{S a}_{4}$, Theorem 0.1], the inclusion $|K|_{m} \subset \overline{|K|}^{l_{1}}$ is a fine homotopy equivalence. Then it follows that $A$ is a $Z$-set in $|K|_{m}$. By the result of $\left[\mathbf{S a}_{3}\right], K$ is a combinatorial $\infty$-manifold. This completes the proof.

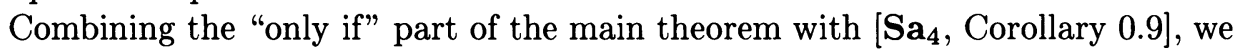
have

2.3 COROLLARY. For a combinatorial $\infty$-manifold $K, \overline{|K|}^{l_{1}}$ is an $l_{2}$-manifold which contains $|K|_{m}$ as an f.d. cap set.

Thus Conjecture 0.8 in $\left[\mathbf{S a}_{4}\right]$ is true. By Henderson $[\mathbf{H e}], l_{2}$-manifolds are topologically classified by homotopy type. Then from the topological uniqueness of f.d. cap sets for an $l_{2}$-manifold $\left[\mathbf{C h}_{1}\right]$, we have

2.4 COROLLARY. If two combinatorial $\infty$-manifold $K$ and $L$ have the same homotopy type, then the pairs $\left(\overline{|K|}^{l_{1}},|K|_{m}\right)$ and $\left(\overline{|L|}^{l_{1}},|L|_{m}\right)$ are homeomorphic.

3. Remarks. For a contractible combinatorial $\infty$-manifold $K, \operatorname{cl}_{Q}|K|$ is homeomorphic to $Q$ by $\left[\mathbf{S a}_{5}\right.$, Corollary 2.3]. As seen in the proof of Theorem 2.2, $t \cdot \overline{|K|}^{Q^{*}}=\left(t \cdot \operatorname{cl}_{Q}|K|\right) \backslash\{0\}$ is a $Z$-set in $\overline{|K|}^{Q^{*}}=\left(\operatorname{cl}_{Q}|K|\right) \backslash\{0\}$ for $0<t<1$. Since $\{0\}$ is a $Z$-set in $\operatorname{cl}_{Q}|K|$, it follows that $t \cdot \operatorname{cl}_{Q}|K|$ is a $Z$-set in $\operatorname{cl}_{Q}|K|$ for $0<t<1$. Then as before, we can prove that

$$
\operatorname{cl}_{Q}|K| \backslash \mathrm{cl}_{S_{1}^{+}}|K|=\left(\operatorname{cl}_{Q}|K|\right) \backslash \overline{|K|}^{l_{1}}
$$

is a cap set for $\operatorname{cl}_{Q}|K|$. Thus we have

3.1 PRoposition. For a contractible combinatorial $\infty$-manifold $K$, the pair $\left(\operatorname{cl}_{Q}|K|, \operatorname{cl}_{S_{1}^{+}}|K|\right)=\left(\mathrm{cl}_{Q}|K|, \overline{|K|}^{l_{1}}\right)$ is homeomorphic to $(Q, s)$.

In connection with our results, the following general problem is very interesting.

3.2 PROBLEM. Let $M$ be an $l_{2}^{f}$-manifold with a metric d. Under what condition is the completion of the metric space $(M, d)$ an $l_{2}$-manifold?

\section{REFERENCES}

[An] R. D. Anderson, On sigma-compact subsets of infinite-dimensional spaces, unpublished manuscript.

[Ch $\mathbf{C h}_{1}$ T. A Chapman, Dense sigma-compact subsets of infinite-dimensional manifolds, Trans. Amer. Math. Soc. 154 (1971), 399-426.

[Ch2] _ Lectures on Hilbert cube manifolds, CBMS Regional Conf. Ser. in Math., no. 28, Amer. Math. Soc., Providence, R.I., 1976.

[He] D. W. Henderson, Infinite-dimensional manifolds are open subsets of Hilbert space, Bull. Amer. Math. Soc. 75 (1969), 759-762.

[Hu] S.-T. Hu, Theory of retracts, Wayne State Univ. Press., Detroit, Mich., 1965. 
[Sa $]$ K. Sakai, Combinatorial infinite-dimensional manifolds and $\mathbf{R}^{\infty}$-manifolds, Topology Appl. 26 (1987), in press.

[Sa $\left.\mathbf{S a}_{2}\right]$, On topologies of triangulated infinite-dimensional manifolds, J. Math. Soc. Japan 39 (1987), in press.

[Sas] _ Simplicial complexes triangulating infinite-dimensional manifolds, preprint.

[Sa $\left.\mathbf{S a}_{4}\right]$ - Completions of metric simplicial complexes by using $l_{p}$-norms, Topology Proc. 11 (1986).

$\left[\mathbf{S a}_{5}\right]-, A Q$-manifold local-compactification of a metric combinatorial $\infty$-manifold, Proc. Amer. Math. Soc. 100 (1987), in press.

[To] H. Toruńczyk, On CE-images of the Hilbert cube and characterization of Q-manifolds, Fund. Math. 106 (1980), 31-40.

Institute of Mathematics, University of TSUKUba, SakURA-MURA, Ibaraki, 305 JAPAN (Current address)

Department of Mathematics, Louisiana State University, Baton Rouge, LOUISIANA 70803 